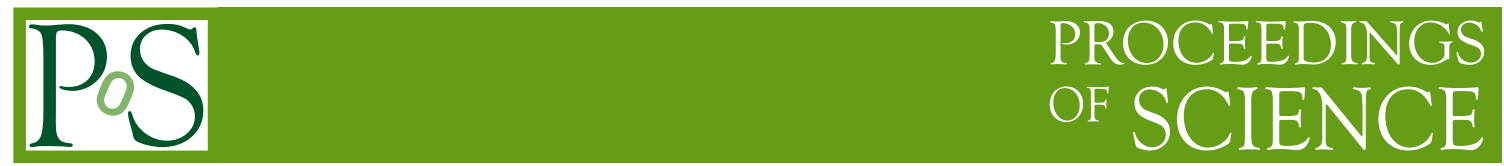

\title{
Jet cross sections in photoproduction at ZEUS
}

\section{Denys Lontkovskyi*}

On behalf of the ZEUS collaboration

Taras Shevchenko National University of Kyiv,

Bogolyubov Institute for Theoretical Physics,

E-mail: denys.lontkovskyi@desy.de

Precise measurements of single- and double-differential inclusive-jet cross sections in photoproduction from the ZEUS collaboration are presented. Next-to-leading-order QCD calculations give a reasonable description of the data. A new precise value of the strong coupling was extracted from the measurements. In addition, the measurements have the potential to constrain the parton distribution functions in the photon and the proton.

XVIII International Workshop on Deep-Inelastic Scattering and Related Subjects, DIS 2010 April 19-23, 2010

Firenze, Italy

${ }^{*}$ Speaker. 


\section{Introduction}

Photoproduction of jets is the main source of jets at the $e p$ collider HERA. Two processes contribute at lowest order of perturbative QCD (pQCD) to the photoproduction of jets. These are the direct process, in which the quasi-real photon directly couples to the partons in the proton; and the resolved process, in which the photon exhibits hadronic structure. The cross section for jet production in PQCD may be written as the convolution of the flux of quasi-real photons, which is estimated using the Weizsäker-Williams approximation, the photon and proton parton density functions (PDFs) and the partonic cross section which can be calculated in pQCD.

Inclusive-jet cross sections in photoproduction were measured using the ZEUS detector. The measurements were used to make a stringent test of next-to-leading order (NLO) $\mathscr{O}\left(\alpha_{s}^{2}\right)$ pQCD predictions. A value of the strong coupling, $\alpha_{s}$, was extracted from the measurements and its energy-scale dependence was determined. In addition, these precise cross-section measurements have the potential to constrain the proton and photon PDFs.

\section{Single- and double-differential inclusive-jet cross sections}

The cross sections were measured using $189 \mathrm{pb}^{-1}$ of ZEUS data [1]. The phase space of the measurement is given by the virtuality of the photon $Q^{2}<1 \mathrm{GeV}^{2}$ and the inelasticity $0.2<y<$ 0.85 . Jets were reconstructed using the $k_{T}$ cluster algorithm [2] in the longitudinally invariant inclusive mode [3] in the laboratory frame and required to have $E_{T}^{\text {jet }}>17 \mathrm{GeV}$ and $-1<\eta^{\text {jet }}<2.5$. Figures $1 \mathrm{a}$ ) and b) show the cross sections as functions of $E_{T}^{\text {jet }}$ and $\eta^{\text {jet }}$, respectively. The $\frac{d \sigma}{d E_{T}^{j e t}}$ measured cross section is a steeply falling function which decreases about five orders of magnitude within the measured range. The cross section $\frac{d \sigma}{d \eta^{j e t}}$ presents a peak at $\eta^{j e t} \approx 0.7$. In addition, inclusive-jet cross sections as functions of $E_{T}^{\text {jet }}$ for different $\eta^{\text {jet }}$ regions were measured (see figure $2)$. The jet spectrum becomes harder as $\eta^{\text {jet }}$ increases. The data have small experimental uncertainties, which amount to $\pm 2 \%$ ( $\pm 7 \%$ ) for low- (high-) $E_{T}^{\text {jet }}$ and $\pm 4 \%$ ( $\pm 8 \%$ ) for low- (high-) $E_{T}^{\text {jet }}$ for uncorelated and corelated jet-energy scale uncertainties, respectively. Next-to-leading-order calculations were performed using the program by M. Klasen, T. Kleinwort and G. Kramer [4] with renormalisation and factorisation scales $\mu_{R}=\mu_{F}=E_{T}^{j e t}$, ZEUS-S for the proton PDFs and GRV-HO for the photon PDFs. The predictions describe well both the shape and normalisation of the data except for the low- $E_{T}^{j e t}$ and high- $\eta^{j e t}$ regions. This discrepancy might be explained, as shown in figure 3, by non-perturbative effects, which are not included in the calculations but simulated with the PYTHIA 6.1 Monte Carlo (MC) generator [5]. The non-perturbative effects increase the jet rate at high $\eta^{\text {jet }}$ (see figure 3 a). It may also indicate an inadequacy of the photon PDFs. The discrepancy disappears for $E_{T}^{\text {jet }}>21 \mathrm{GeV}$ (see figure $3 \mathrm{~b}$ ).

Several sources of theoretical uncertainty were estimated. The dominant sources are those arising from the terms beyond NLO and from the uncertainty due to the proton PDFs, which is significant at high- $E_{T}^{j e t}$. The uncertainty due to terms beyond NLO was estimated by varying the renormalisation scale by factors 2 and 0.5 . The proton-PDFs contribution was estimated by using 22 additional sets from the ZEUS-S analysis. The uncertainty due to the modelling of the hadronisation and parton-shower effects was estimated by using different MC generators. The uncertainty from the photon PDFs was estimated by using an alternative set. The uncertainty due 
to $\alpha_{s}$ was also estimated. The theoretical uncertainties as functions of $E_{T}^{\text {jet }}$ and $\eta^{\text {jet }}$ are presented in figure 4.

\section{Determination of $\alpha_{s}$}

The procedure to determine $\alpha_{s}$ is based on the $\alpha_{s}$ dependence of the pQCD calculations, taking into account the correlation with the PDFs. Calculations were performed for different sets of the proton PDFs. Different values of $\alpha_{s}$ were assumed for each calculation, consistent with the value assumed in the PDFs. The NLO dependence on $\alpha_{s}\left(M_{Z}\right)$ was parametrised by a parabola. Finally, a value of $\alpha_{s}$ was obtained from the measured observable using this parametrisation. This procedure handles correctly the complete $\alpha_{s}$-dependence of the NLO calculations in the fit while preserving the correlation between $\alpha_{s}\left(M_{Z}\right)$ and the PDFs

The value of $\alpha_{s}\left(M_{Z}\right)$ determined for $21 \mathrm{GeV}<E_{T}^{\text {jet }}<71 \mathrm{GeV}$ is

$$
\alpha_{s}\left(M_{Z}\right)=0.1208_{-0.0023}^{+0.0024}(\exp .)_{-0.0033}^{+0.0044}(\text { th. })
$$

. The experimental uncertainties are dominated by the jet-energy scale $\left({ }_{-1.9}^{+2.0} \%\right)$. The theoretical uncertainties are dominated by those arising from the terms beyond NLO $\left({ }_{-2.5}^{+2.4} \%\right)$ and the photon PDFs $(+2.4 \%)$. The measured energy-scale dependence of the coupling is presented in figure 5 and is in good agreement with the predicted running of $\alpha_{s}$.

\section{Summary}

New measurements of inclusive-jet cross sections in photoproduction were presented. Perturbative NLO calculations describe the data well except at low $E_{T}^{j e t}$ and high $\eta^{\text {jet }}$. This may indicate the possible presence of non-perturbative effects or an inadequacy of the photon PDFs. The highprecision data may constrain further the proton and photon PDFs if used together with inclusive data in the QCD fits to extract the parametrisations. The new precise value of the strong coupling obtained is consistent with other determinations at HERA and with the world average. The measured energy-scale dependence of the coupling is in good agreement with the predicted running of $\alpha_{s}$ over a wide range in $E_{T}^{j e t}$.

\section{References}

[1] ZEUS collaboration, ZEUS-prel-10-003.

[2] S. Catani et al., Nucl. Phys. B 406, 187 (1993).

[3] S.D. Ellis and D.E. Soper, Phys. Rev. D 48, 3160 (1993).

[4] M. Klasen, T. Kleinwort, and G. Kramer, Eur. Phys. J. Direct C 1, 1 (1998).

[5] T. Sjöstrand, Comp. Phys. Comm. 82, 74 (1994). 


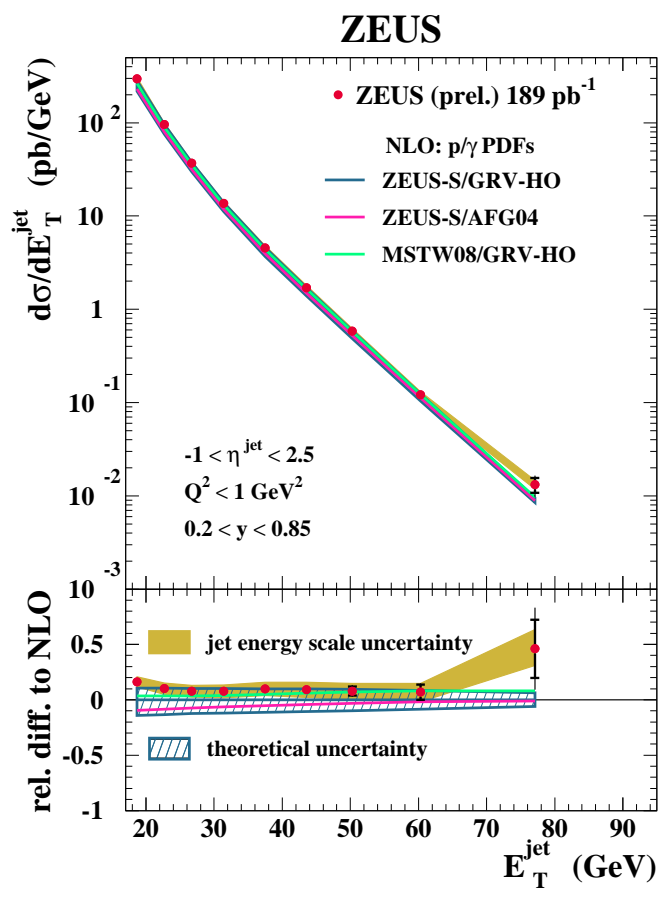

(a)

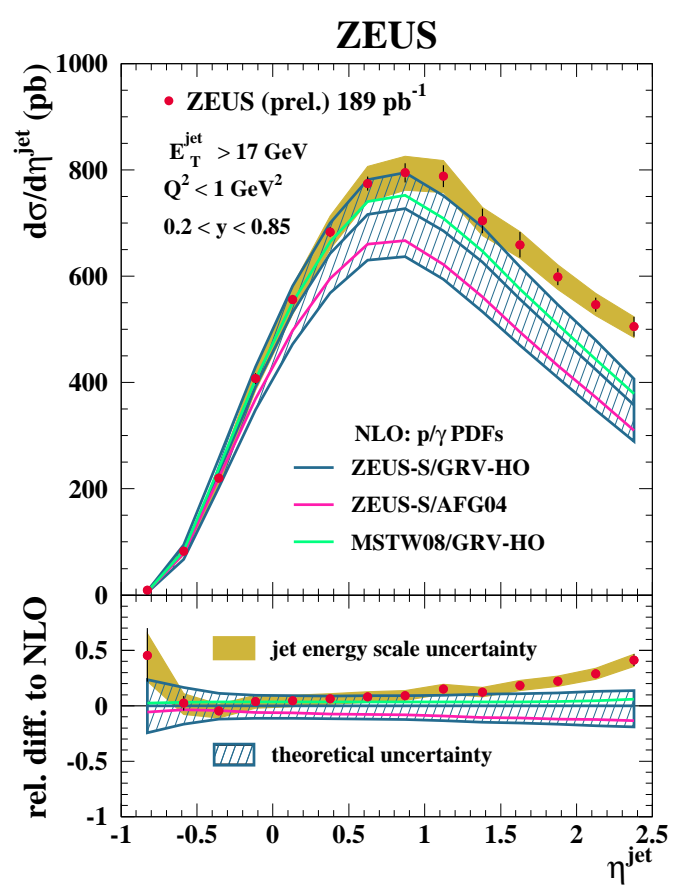

(b)

Figure 1: The inclusive-jet cross sections as functions of (a) $E_{T}^{j e t}$ and (b) $\eta^{j e t}$.

\section{ZEUS}

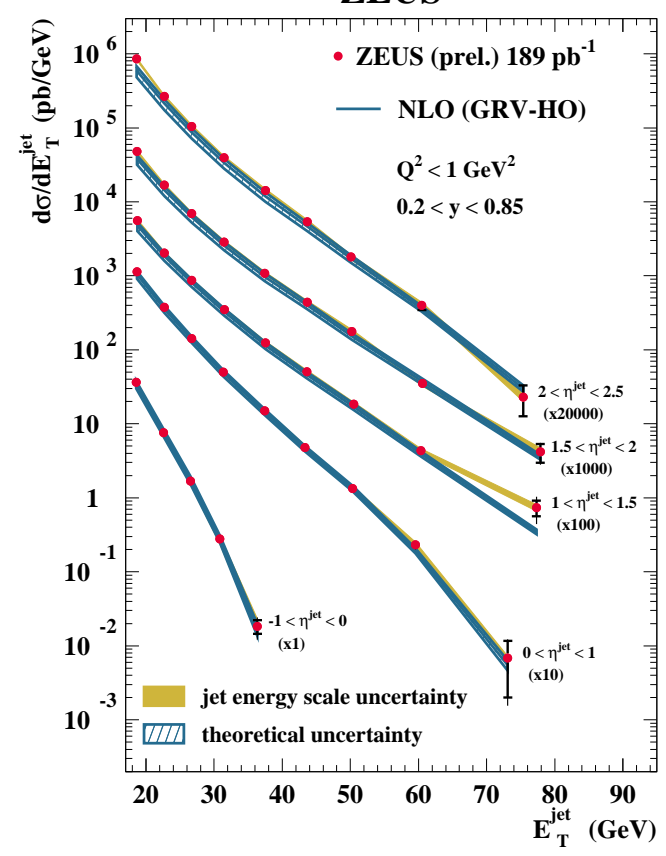

(a)

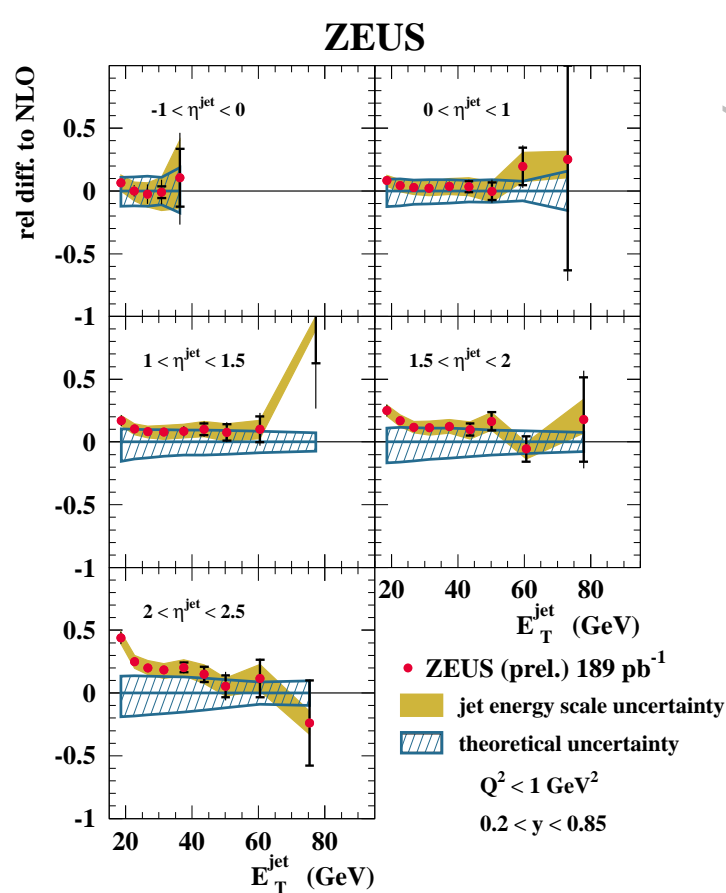

(b)

Figure 2: The inclusive-jet cross sections as functions of $E_{T}^{\text {jet }}$ for different regions of $\eta^{\text {jet }}$ (a). The relative difference between the data and the NLO calculations (b) 


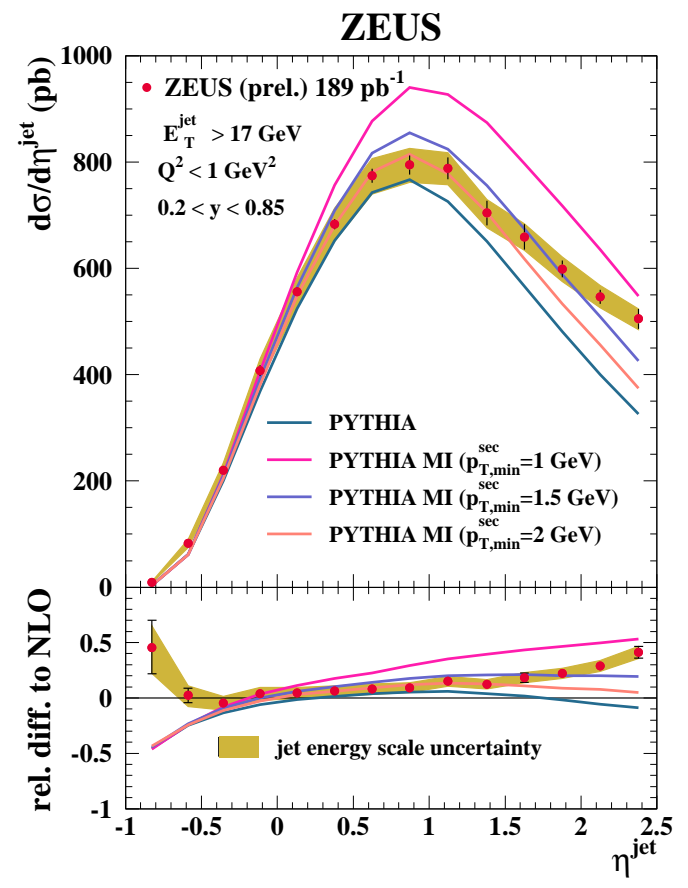

(a)

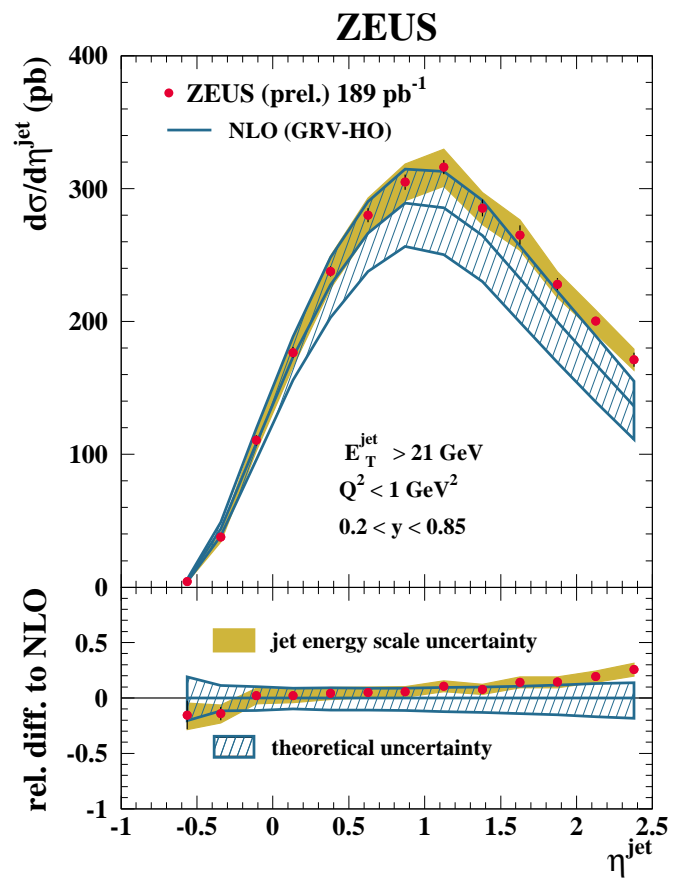

(b)

Figure 3: The inclusive-jet cross sections as functions of $\eta^{\text {jet }}$ (a) for jets with $E_{T}^{\text {jet }}>17 \mathrm{GeV}$ compared with the NLO predictions corrected to include non-perturbative effects and (b) for jets with $E_{T}^{\text {jet }}>21 \mathrm{GeV}$.

\section{ZEUS}

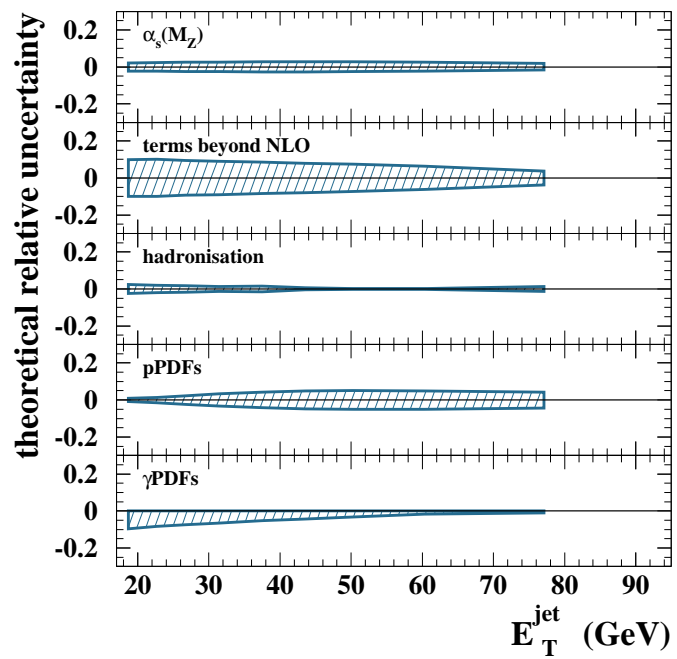

(a)

\section{ZEUS}

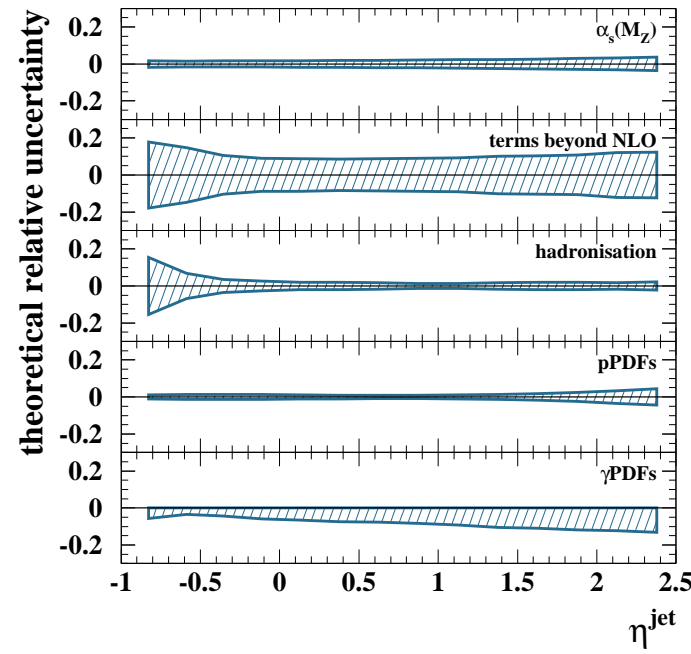

(b)

Figure 4: The theoretical uncertainties as functions of (a) $E_{T}^{j e t}$ and (b) $\eta^{j e t}$. 


\section{ZEUS}

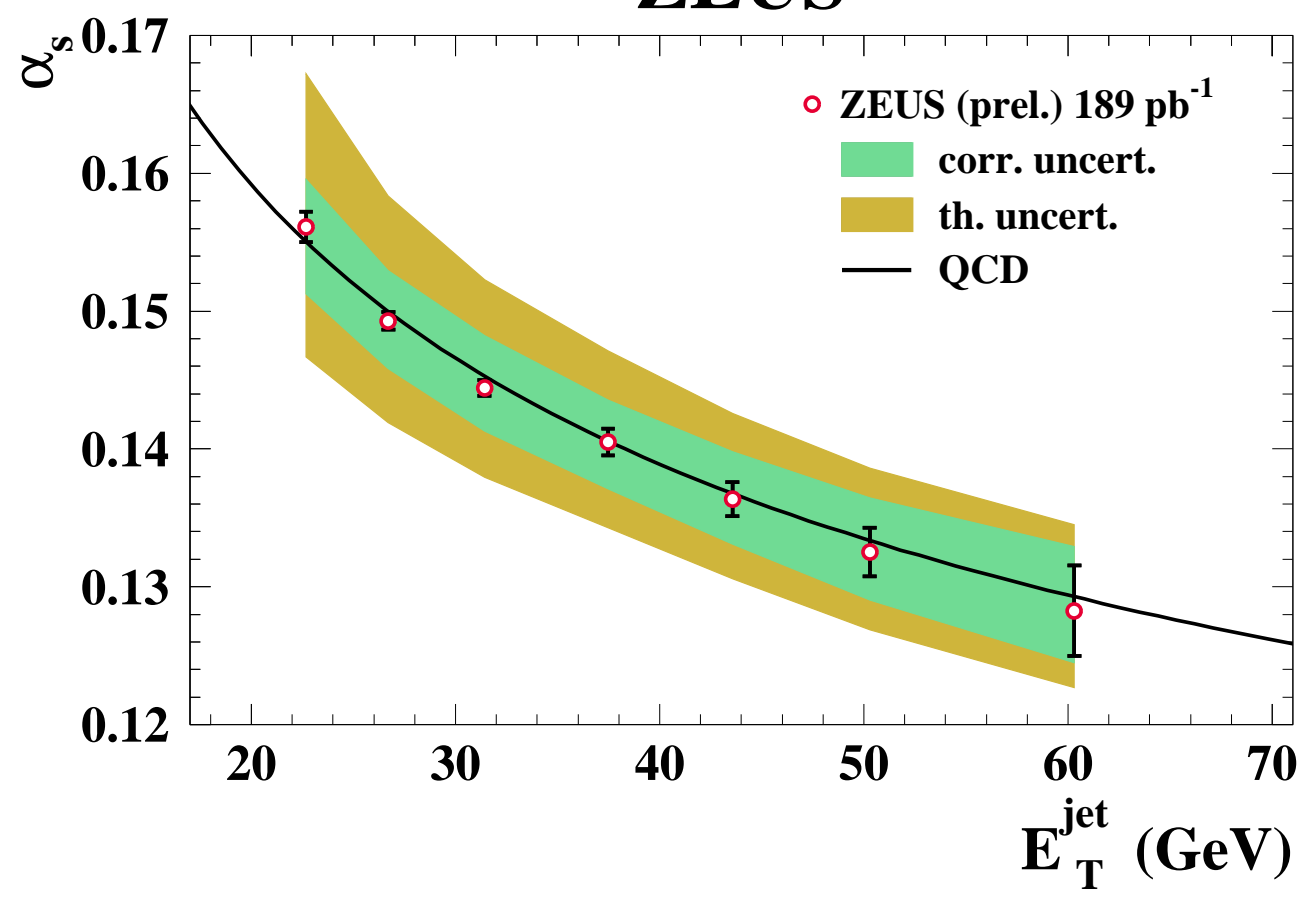

Figure 5: The energy-scale dependence of $\alpha_{s}$ 